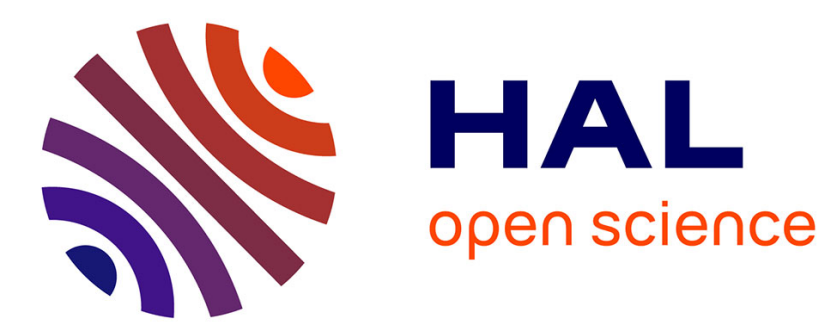

\title{
Faire la révolution dans les confins caucasiens
}

Etienne Peyrat

\section{To cite this version:}

Etienne Peyrat. Faire la révolution dans les confins caucasiens: La liberté côté cour et côté jardin. Vingtième siècle. Revue d'histoire, 2017, 1917, un moment révolutionnaire, 135, pp.56-72. 10.3917/ving.135.0056 . halshs-01783399

\section{HAL Id: halshs-01783399 \\ https://shs.hal.science/halshs-01783399}

Submitted on 7 May 2018

HAL is a multi-disciplinary open access archive for the deposit and dissemination of scientific research documents, whether they are published or not. The documents may come from teaching and research institutions in France or abroad, or from public or private research centers.
L'archive ouverte pluridisciplinaire $\mathbf{H A L}$, est destinée au dépôt et à la diffusion de documents scientifiques de niveau recherche, publiés ou non, émanant des établissements d'enseignement et de recherche français ou étrangers, des laboratoires publics ou privés. 


\title{
Faire la révolution dans les confins caucasiens en 1917
}

La liberté côté cour et côté jardin

\author{
Étienne Forestier-Peyrat
}

Comment la révolution de 1917 a-t-elle gagné les confins de l'Empire tsariste alors tenus par l'armée russe ? À partir d'une synthèse des situations de l'Iran du Nord et de l'Anatolie orientale, l'auteur montre qu'il n'y a pas eu diffusion de la révolution, mais construction locale des enjeux révolutionnaires. Outre la porosité préalable des frontières, l'appropriation de la révolution dans ces territoires lointains a pu compter sur l'interprétation commune du passé de la région, notamment des révolutions des années 1900, et sur la conversion de la révolution russe en mouvement local d'opposition des populations à l'autorité de leurs consuls. Parce qu'il montre l'esquisse d'une solidarité transnationale, avortée dans les faits, l'article donne une autre intellection, plus dense et plus subtile, des processus révolutionnaires.

Les processus révolutionnaires, comme tout événement politique d'ampleur, bousculent les clivages sociaux, économiques et politiques, produisant de nouveaux partages le long des fractures qu'ils ouvrent. Les frontières intérieures, qui rejettent dans l'ancien régime certaines franges de la société et mettent à l'honneur les proscrits de la veille, sont manifestes. Mais les révolutions donnent aussi lieu à une autre forme de délimitation, spatiale, entre un intérieur et un extérieur de la révolution. D'un côté, des populations prises, volontairement ou non, dans le processus révolutionnaire. De l'autre, des populations qui peuvent assister à la révolution de loin, en spectatrices, ou même y rester indifférentes ${ }^{1}$. Les frontières politiques de l'État où se déclenche la révolution sont des candidates naturelles pour en définir l'enveloppe extérieure, mais elle n'ont rien d'évident, en particulier lorsqu'elles ne correspondent que partiellement à des découpages linguistiques, culturels, religieux ou économiques.

Comment définir les frontières d'une révolution, alors même que ses dynamiques internes ne se réduisent nullement à un gradient entre capitale et province, où des processus connectés mais distincts s'observent? Ces variations régionales justifient le regain d'approches spécifiques des révolutions provinciales, particulièrement riches dans le cas d'une révolution

\footnotetext{
${ }^{1}$ Monique Cubells (dir.), La Révolution française : la guerre et la frontière, Paris, Comité des travaux historiques et scientifiques, 2000.
} 
de 1917 qui se projette aux dimensions de l'Eurasie, dans un empire fragilisé par l'effort de guerre $^{2}$. Les discours révolutionnaires eux-mêmes, en définissant le ou les peuples politiques auxquels ils s'adressent, ont la capacité de suggérer les frontières qu'ils veulent avoir, mais ils peuvent aussi recevoir des échos inattendus en dehors du public qu'ils se donnent. Vue de France, la révolution de février 1917 apparaît comme un processus lointain, en dépit de son impact concret sur la guerre et sur certains acteurs sociopolitiques ${ }^{3}$. Le problème est plus difficile à dénouer si l'on se place sur les frontières de l'Empire tsariste, en ce début de 1917. Jusqu'où s'étend spatialement le processus révolutionnaire ? Depuis 1914, des territoires ont été conquis, perdus, les frontières de l'Empire se sont déplacées et plusieurs de ces confins impériaux se trouvent dans des situations ambiguës au point de vue politique et juridique, à l'image d'un Iran du Nord et d'une Anatolie orientale ottomane où stationnent les troupes russes. La mobilisation économique et humaine de la guerre a elle-même produit de nouvelles formes d'interaction, de l'Europe centrale à l'Extrême-Orient, entre sujets des tsars et populations limitrophes ${ }^{4}$. L'objectif de cet article est de réfléchir, en partant de l'exemple des zones-frontières du Caucase tsariste, aux appropriations auxquelles peut donner lieu la révolution dans ces territoires incertains.

On voudrait ici montrer que la manière dont se déroule la révolution dans les territoires marginaux de l'empire révèle l'existence d'espaces politiques à cheval sur les entités étatiques. Facilitée par des échanges régionaux intenses, par la présence de communautés linguistiques, nationales et religieuses transfrontalières, cette existence découle aussi de l'histoire mouvementée de la décennie précédant 1917. La presse tsariste, arménophone, turcophone ou russophone, circule largement dans les villes du Nord de l'Iran, comme le rappelle une carte réalisée à la veille de la révolution de Février par le Messager arménien édité à Moscou, qui représente sous la forme de faisceaux rayonnants la diffusion de ce journal vers le Moyen-Orient ${ }^{5}$. S'engageant dans ces interstices, la révolution donne lieu à des échos et à des appropriations au-delà de l'espace tsariste stricto sensu. Les frontières

\footnotetext{
${ }^{2}$ Donald J. Raleigh, Revolution on the Volga : 1917 in Saratov, Ithaca, Cornell University Press, 1986; Orlando Figes, Peasant Russia, Civil War: The Volga Countryside in Revolution, 1917-1921, Oxford, Clarendon Press, 1989.

${ }^{3}$ Marc Ferro, L'Occident devant la révolution soviétique: l'histoire et ses mythes, Bruxelles, Complexe, 1980.

${ }^{4}$ Chia Yin Hsu, "The "Color" of Money: The Ruble, Competing Currencies, and Conceptions of Citizenship in Manchuria and the Russian Far East, 1890s-1920s », The Russian Review, 73, 2014, p. 93-94.

${ }^{5}$ « Karta rasprostranenija “Armjanskogo Vestnika” », Armjanskij Vestnik, 6, 5 février 1917 (18 février 1917), p. 7.
} 
politiques de l'ancien empire se trouvent temporairement brouillées, alors que s'esquisse un processus révolutionnaire qui s'appuie sur des phénomènes de traduction politique et de déplacement. L'exemple des confins caucasiens souligne cependant l'importance, d'abord productive, de certains malentendus, qui deviennent problématiques à mesure que le délitement du pouvoir russe y crée des tensions politiques, mais aussi sociales et économiques, et fait finalement obstacle au développement transfrontalier d'une révolution.

\section{Une révolution russe sur un front militaire ?}

Le Caucase dans lequel arrive la nouvelle des événements de Petrograd des 23 au 28 février (8 au 13 mars) 1917 n'est plus ce qu'il était ${ }^{6}$. Officiellement, les frontières de la vice-royauté du Caucase avec l'Empire ottoman et l'Iran restent certes identiques à leur tracé d'avantguerre. L'armée tsariste occupe pourtant de vastes territoires sur le sol de ces deux États. La présence de l'armée russe en Iran remonte à décembre 1911, lorsqu'elle intervient pour écraser définitivement le mouvement constitutionnel persan dont les bastions se trouvent dans le Nord du pays, aux portes de la Russie - dans les provinces d'Azerbaïdjan, du Gilan et du Mazandaran ${ }^{7}$. Si les autorités persanes ne sont pas évincées, l'autorité de fait se déplace dans la région vers les commandants militaires et les consuls russes, qui reçoivent leurs directives du ministère des Affaires étrangères et de la vice-royauté du Caucase, sise à Tiflis (actuelle Tbilissi). À ce protectorat informel sur l'Iran du Nord s'ajoute, à partir de l'année 1915, une politique d'occupation liée à la guerre mondiale en Anatolie orientale. Une série d'offensives victorieuses de la Russie débouchent, en 1915-1916, sur l'annexion d'une grande partie de la région. À la mi-février 1916, la ville d'Erzurum tombe aux mains des Russes. Cette avancée à l'intérieur des terres se trouve complétée par une progression sur le littoral, où les Russes prennent le port de Trabzon, le 18 avril $1916^{8}$. La série des défaites se prolonge jusqu'au début du mois d'août 1916, lorsque les villes de Bayburt et d'Erzincan tombent, posant les bases les plus avancées de la présence russe ${ }^{9}$.

\footnotetext{
${ }^{6}$ Ronald Grigor Suny, The Soviet Experiment : Russia, the USSR and the Successor States, Oxford, Oxford University Press, 1998, p. 36-38.

${ }^{7}$ James D. Clark, « Constitutionalists and Cossacks : The Constitutional Movement and Russian Intervention in Tabriz, 1907-1911 », Iranian Studies, 39 (2), 2006, p. 199-225.

8 Nikolaj Novikov, Operatsii flota protiv berega na Chernom more v 1914-1917 gg., Moscou, Gosvoenizdatel'stvo, 1937, p. 186-237.

${ }^{9}$ Ulrich Trumpener, « The Turkish War, 1914-1918», in John Horne (dir.), A Companion to World War I, Chichester, Blackwell Publishing, 2010, p.100 ; Nikolaj Korsun, Pervaja mirovaja vojna na Kavkazskom fronte :
} 
La guerre a renforcé la porosité entre Caucase russe, Iran du Nord et provinces ottomanes occupées, du fait de circulations militaires, humaines, économiques et administratives ${ }^{10}$. D'expression de la puissance russe, une telle situation peut aisément se retourner en faiblesse, comme tentent de le faire valoir les autorités de la vice-royauté caucasienne dans les premiers jours de la révolution, pour essayer d'en limiter l'impact. Une circulaire du 3 mars (16 mars) de l'adjoint du vice-roi aux affaires civiles, le prince Orlov, appelle à assurer la continuité de l'État dans ce « théâtre d'actions militaires, où nos troupes ont entrepris et poursuivent avec éclat une offensive sur le front persan ${ }^{11} »$. L'agitation qui se saisit des garnisons et unités du front dans les jours qui suivent l'annonce de la révolution ne peut qu'inquiéter l'état-major caucasien. À la forteresse de Kars, le général Lindenbaum et ses adjoints sont arrêtés par les soldats ${ }^{12}$. Sur le front même, «tout le monde se réjouit» des événements de Petrograd, comme l'écrit le sous-lieutenant Loparev du front d'Erzincan, le 4 mars (17 mars) ${ }^{13}$.

Les militaires sont les premiers concernés par l'extraterritorialité de la révolution, comme l'illustre une photographie prise par l'infirmière Nino Djordjadze, de la Croix-Rouge russe, dans la localité de Hınıs, en Anatolie occupée. On y voit la proclamation le 4 mars 1917 (17 mars 1917) du gouvernement provisoire, devant la foule des soldats russes assemblés (document 2) ${ }^{14}$. Les unités militaires, où qu'elles se trouvent, sont associées au processus révolutionnaire par la création de soviets subordonnés au soviet régional de Transcaucasie, dirigé par le menchevique géorgien Noé Jordania. Ce soviet entre rapidement en concurrence avec le Comité spécial caucasien (Ozakom) établi le 5 mars (18 mars) par le gouvernement provisoire pour remplacer la vice-royauté ${ }^{15}$. Composé de membres caucasiens de la Douma, revenus de Petrograd, et de représentants des principaux partis du Caucase, l'Ozakom ne

operativno-strategicheskij ocherk, Moscou, Voennoe Izdatel'stvo Ministerstva Vooruzhennykh Sil SSSR, 1946, p. 63-70.

${ }^{10}$ Halit Dündar Akarca, «Imperial Formations in Occupied Lands: The Russian Occupation of Ottoman Territories during the First World War », thèse de doctorat, Université de Princeton, 2014.

${ }^{11}$ Circulaire du prince Orlov aux gouverneurs, 3 mars 1917 (16 mars 1917), reproduite dans S.E. Sef, Revoljutsija 1917 goda v Zakavkaz’i (Dokumenty, materialy), Tiflis, Zakkniga, 1927, p. 57-59.

${ }^{12}$ Michael S. Frenkin, Russkaja armija i revoljutsija, 1917-1918, Munich, Logos, 1978, p. 58-59.

${ }^{13}$ Ibid., p. 122 ; A. Metelev, « Fevral' v Karse », Proletarskaja Revoljutsija, 1 (13), 1923, p. 227-234.

${ }^{14}$ Giorgi Kalandia, Kartvlebi p’irvel msoplio omshi, Tbilissi, Sakartvelos Sakhemts'ipo Muzeumi, 2014, p. 88-89.

${ }^{15}$ Procès-verbal du gouvernement provisoire, 5 mars 1917 (18 mars 1917), in Boris Dodonov (dir.), Arkhiv novejshej istorii Rossii: Zhurnaly zasedanij Vremennogo pravitel'stva, Mart-oktjabr' 1917, Moscou, ROSSPEN, 2001, t. VII, p. 35-36. 
parvient pas à affirmer son pouvoir du fait d'une légitimité politique faible et de problèmes organisationnels certains ${ }^{16}$. La chute de la vice-royauté et le départ du grand-duc Nikolaï Nikolaïevitch qui la dirigeait signifient en effet un fort étiolement des institutions exécutives et administratives dans la région ${ }^{17}$.

\section{Intérieur et extérieur de la révolution}

La photographie de Nino Djordjaze vaut autant par ce qu'elle montre que ce par ce qui n'y entre pas. La foule compacte des soldats de l'armée russe ne permet en effet guère d'y discerner la présence éventuelle d'habitants de la localité. Si les militaires et les civils russes présents en Anatolie orientale et en Iran du Nord sont concernés au premier titre par la révolution, les populations locales n'y restent pourtant pas indifférentes. Dans ses mémoires, Şeref Tipi, alors enfant à Erzurum, écrit :

«Tante Kürtkızı est venue voir ma mère pour lui raconter quelque chose. De sa petite maison d'un étage, elle pouvait facilement voir le quartier-général des Russes qui se trouvait en face. Elle a dit qu'une grande agitation et des préparatifs avaient lieu dans le camp russe ${ }^{18}$. »

Les autochtones projettent souvent sur cette révolution lointaine les attentes qu'ils fondent sur un changement de leur propre situation. Le flou qui entoure la révolution favorise la diversité de ces projections ${ }^{19}$.

Les télégrammes de félicitations envoyés au gouvernement provisoire révèlent l'existence d'un espace révolutionnaire frontalier, sans solution de continuité. Télégrammes du Caucase russe, des garnisons tsaristes sur le front, des communautés russes en Iran du Nord et des

16 Sur la prolifération d'institutions municipales, citoyennes, politiques, dans les premiers jours de la révolution, voir S. E. Sef, Revoljutsija 1917..., op. cit., 1927, p. 62-73; aucun groupe politique caucasien n'est véritablement satisfait du melting pot qu'est l'Ozakom : le cadet russe Kharlamov, le menchevique Tchkhenkeli, le social-fédéraliste Abashidze, le musulman Djafarov et le cadet Papadjanian. Sur le mécontentement des Dachnaks, voir Anahide Ter Minassian, La République d'Arménie, 1918-1920, Bruxelles, Complexe, 1989, p. $17-18$.

${ }^{17}$ Morgan Philips Price, War and Revolution in Asiatic Russia, Londres, Allen \& Unwin, 1918, p. 286 ; sur le départ en mode mineur du grand-duc, escorté par des drapeaux rouges et La Marseillaise à la gare de Tiflis, voir Morgan Philips Price, «The Background of the Revolution », Manchester Guardian, 11 avril 1917, reproduit dans Morgan Philips Price et Tania Rose (dir.), Dispatches from the Revolution : Russia, 1916-1918, Durham, Duke University Press, 1997, p. 33-34.

${ }^{18}$ Şeref Tipi, Pışıbba (1860-1926), Istanbul, Remzi Kitabevi, 2005, p. 196.

${ }^{19}$ Lev Bykovs'kyj, Na Kavkaz'ko-turets 'komu fronti. Spomyny z 1916-1918 rr., Winnipeg-Denver, Research Institute of Volhyn, 1968, p. 58 ; Isaak Izrailevich Mints (dir.), Pobeda Sovetskoj vlasti v Zakavkaz'e, Tbilissi, Metsniereba, 1971, p. 75. 
populations locales se mêlent dans un même flot ${ }^{20}$. Le 20 mars 1917, le Premier ministre iranien Vosûq ed-Dovleh envoie au gouvernement russe un télégramme saluant la révolution et, le 2 avril, soixante et onze membres de l'ancien Madjlis prennent l'initiative d'écrire à la Douma, retrouvant les échanges télégraphiques de l'époque des révolutions constitutionnelles dans les années $1900^{21}$. Dans la rapidité et les formes de cette réaction, les liens antérieurs avec la Russie, sa diplomatie ou ses formes politiques, jouent un rôle significatif. Les groupes minoritaires des territoires occupés d'Anatolie orientale et du Nord de l'Iran, cultivés par la diplomatie russe depuis le $19^{\mathrm{e}}$ siècle, sont particulièrement réceptifs à un changement dont ils espèrent une amélioration pour eux-mêmes. À la fin du mois de mars, les autorités ecclésiastiques des Assyriens multiplient les appels au gouvernement provisoire ${ }^{22}$. Dans une supplique au prince Lvov, l'évêque assyrien de la ville de Khoy, Ilia, rappelle les malheurs subis par ses ouailles lors de la guerre et exprime le souhait que les Assyriens « ne [soient] pas oubliés au moment de la victoire décisive de la Russie libre contre l'Allemagne, ennemie de l'autodétermination des peuples ${ }^{23}{ }^{\prime}$. Ilia reprend dans cette lettre l'idée que la révolution met fin à l'anomalie politique tsariste, élevant enfin la Russie au rang des démocraties européennes $^{24}$.

Dans ces cercles concentriques de participation à la révolution, le front représente un obstacle certain. Moins du fait de son existence, en réalité, que parce qu'il est fuyant et qu'il n'y a pas en 1916, pour reprendre les observations d'Aleksej Tatischev, de « ligne continue,

${ }^{20}$ Télégramme de la garnison d'Enzeli au prince Lvov, 15 avril 1917 (18 avril 1917) ; GARF, f. 1788, op. 2 , d. 54, 1.4 ; GARF, f. 1788, op. 2, d. 54, 1. 8, télégramme du comité exécutif des Russes de Téhéran à la Douma d'État, 24 avril 1917 (27 mai 1917); Rex A. Wade, The Russian Revolution, 1917, Cambridge, Cambridge University Press, 2000, p. 49-51.

${ }^{21}$ Mikhail Rodzjanko, président de la Douma, répond le 30 avril par le même canal. Mohammad Gholi Majd, Persia in World War One and Its Conquest by Great Britain, Lanham, University Press of America, 2003, p. 141-142 ; une mission présidée par Sayyid Ziya ed-Din Tabatabai se rend en Russie pour y étudier la nouvelle situation politique: 'Alî Akbar Velâyatî, Târîkh-e ravâbit-e khâredjî-e Irân dar dovre-ye avval-e Mashrute, Téhéran, Vezârat-e omûr-e khâredje, 1374 [1995-1996], p. 44-45.

${ }^{22}$ Lev Sargizov, Assirijtsy stran Blizhnego i Srednego Vostoka : pervaja chetvert' XX veka, Erevan, Ajastan, 1979, p. 68-69.

${ }^{23}$ Archives centrales d'histoire contemporaine de Géorgie (STsSSA), f. 2081, op. 1, d. 192, 1. 2, télégramme du vice-consul russe à Khoy au prince Lvov, 27 mars 1917 (9 avril 1917).

${ }^{24}$ Cette idée est exprimée de manière célèbre dans le télégramme adressé le 18 avril 1917 ( $1^{\text {er }}$ mai 1917) par le ministre des Affaires étrangères russe, Pavel Miljukov, aux Alliés, qui engage son pays à renforcer l'effort de guerre. Voir Alastair Kocho-Williams, Russia's International Relations in the Twentieth Century, New York, Routledge, 2013, p. 28. 
mais un certain nombre de points stratégiques d'affrontement ${ }^{25} »$. Non qu'aucune interaction ne puisse exister entre les deux camps, comme le rappellent les souvenirs de soldats du front : lorsque de la «musique, des chants et des danses » proviennent du camp adverse, dans le lointain, chacun s'imagine volontiers des choses ${ }^{26}$. La contrebande entre les camps existe elle aussi et voit passer, avant même la révolution, des tracts clandestins. Mais les observations mutuelles directes se font rares, ce qui n'empêche pas la curiosité car les événements de la révolution russe sont vus de manière très indirecte dans l'Empire ottoman, par le biais des agences de presse des puissances centrales ${ }^{27}$. Là où le front est continu, les témoignages ottomans soulignent l'intérêt pour le processus révolutionnaire en cours dans le camp d'en face.

Drapeaux blancs et pancartes se mettent à apparaître du côté russe, à destination des Ottomans en mars $1917^{28}$. Dans ses mémoires, l'officier ottoman Vasfi Şensözen, en service sur un secteur de tranchées, rapporte l'événement suivant :

«Un jour, le soldat de garde vient me raconter que des meetings ont lieu derrière le front russe et qu'à l'un d'entre eux un individu est monté sur une butte, a prononcé un discours, et a été applaudi. Je cours aux tranchées pour voir : les sentinelles disent vrai ${ }^{29}$."

On se trouve ici sur la ligne même du front, qui est transformée en espace d'observation de la révolution russe. Les perceptions y sont certes trompeuses, mais le commandement ottoman décide d'essayer de tirer parti de la révolution à ses propres fins. Ainsi, le commandant ottoman Ali Ihsan Pasha recourt à la diffusion de proclamations « Au peuple russe » dans la région de Van, en mai 1917, où elles sont introduites clandestinement par des soldats

${ }^{25}$ Aleksej Tatishchev, Zemli i ljudi : v gushche pereselencheskogo dvizhenija (1906-1921), Moscou, Russkij Put', 2001, p. 239 ; voir la rubrique « Na turetskikh frontakh », Armjanskij Vestnik, 5, 29 janvier 1917 (11 février 1917), p. 12-13.

${ }^{26}$ Faik Tonguç, Souvenirs d'un officier ottoman (1914-1923), trad. du turc par Bruno Élie, Paris, Petra, 2015, p. 166-167.

${ }^{27}$ M. S. Frenkin, Russkaja armija i revoljutsija, op. cit., p. 273 ; sur la réception de la révolution russe de 1917 dans l'Empire ottoman, voir Uygur Kocabaşoğlu et Metin Berge, Bolşevik Ihtilâli ve Osmanlılar, Istanbul, İletişim, 2006, p. 51-61.

${ }^{28}$ Yüksel Nizamoğlu, Vehip Paşa : kahramanlıktan sürgüne, Istanbul, Yitik Hazine Yayınları, 2013, p. 205206.

${ }^{29}$ Vasfi Şensözen, I. Dünya Savaşı Yılları Kafkas Cephesi Anıları, Istanbul, Okuyan Us, 2013, p. 111. Le texte des mémoires contient une ambiguïté, dans la mesure où il parle à la fois de la première révolution russe et de la révolution bolchevique. Il semble que l'auteur ait ici tendance à écraser la chronologie de l'année 1917, faisant de la révolution de février une révolution bolchevique avant l'heure. 
infiltrés ${ }^{30}$. Le front caucasien ne présente cependant pas de traces de fraternisation sérieuse comme on peut en trouver sur le front occidental au même moment ${ }^{31}$. À l'absence d'expérience antérieure de «cohabitation» dans les tranchées, il convient d'ajouter les difficultés matérielles posées par les décalages linguistiques et politiques forts qui réduisent les possibilités de contact entre les armées sur le front caucasien ${ }^{32}$.

\section{Les consuls, figures de l'ancien régime}

Si une logique de diffusion révolutionnaire est bien à l'œuvre dans les confins caucasiens, le développement du processus suppose une insertion dans les réalités politiques et sociales locales. La diversité interne des communautés russes en Iran du Nord, composée aussi bien de Slaves, de groupes provenant du Caucase tsariste et d'Iraniens ayant acquis la citoyenneté russe, facilite la diversification des registres de la révolution ${ }^{33}$. Ces communautés diverses étaient jusqu'en 1917 structurées à la fois par des institutions propres (notamment des assemblées de marchands) et par l'autorité de consuls devenus très puissants du fait de l'occupation russe de la région depuis la fin de $1911^{34}$. L'attaque contre les consuls, visages locaux de l'ancienne monarchie, est un phénomène connu pour bien des révolutions ${ }^{35}$. Alors

${ }^{30}$ Ibid., p. 112 ; M. S. Frenkin, Russkaja armija i revoljutsija, op. cit., p. 274-275 ; Michael Reynolds parle de tracts saluant la « révolution heureuse » (ihtilâl-i mesut), Shattering Empires : The Clash and Collapse of the Ottoman and Russian Empires, 1908-1918, Cambridge, Cambridge University Press, 2011, p. 168.

${ }^{31}$ Là aussi, cependant, les cas de fraternisation (bratanie) restent concentrés au début du printemps et au moment des fêtes de Pâques, avant que le soviet de Petrograd ne les interdise. Voir Marc Ferro, «Le soldat russe en 1917 : indiscipline, pacifisme, patriotisme et révolution », Annales : économies, sociétés, civilisations, 26 (1), 1971, p. 14-39, p. 24 ; les affirmations de l'historien Anatolij Miller sur l'existence d'une fraternisation ne reposent sur aucune référence précise : Anatolij Miller, Ocherki novejshej istorii Turtsii, Moscou-Leningrad, Izdatel'stvo Akademii Nauk SSSR, 1948, p. 78-79.

${ }^{32}$ M. S. Frenkin, Russkaja armija i revoljutsija, op. cit., p. 273.

${ }^{33}$ Morteza Nouraei et Vanessa Martin, « Russian Land Acquisition in Iran from 1828 to 1911 », in Stephanie Cronin (dir.), Iranian-Russian Encounters : Empires and Revolutions Since 1800, Londres, Routledge, 2013, p. $95-107$.

${ }^{34}$ Il semble que les consuls russes en Iran méridional, où ils n’ont jamais exercé des prérogatives similaires, soient moins soumis à ces remises en cause. Voir le témoignage de F. Hale à propos de la révolution parmi les quelques sujets russes de Birjand, en Iran du Sud-Est : F. Hale, From Persian Uplands, Londres, Constable \& Company, 1920, p. 155-157.

${ }^{35}$ Pascal Even (dir.), Les Français à l'étranger et la Révolution, Paris, Ministère des Affaires étrangèresDirection des Archives, 1989 ; Amaury Faivre d'Arcier, Les Oubliés de la liberté : négociants, consuls et missionnaires français au Levant pendant la Révolution (1784-1798), Bruxelles, Peter Lang, 2007, p. $117-118$; 
que les consuls passaient pour des soutiens de la communauté russe dans ses litiges avec les autorités iraniennes ou même des sujets iraniens, les voici mis plus bas que terre dans un renversement qui ne laisse pas d'étonner. Ainsi s'exprime le soviet d'Enzeli dans un rapport de mai 1917 :

«À la différence des consuls européens, qui entrent en contact direct avec leurs ressortissants et avec les négociants locaux, le consul russe est orgueilleux, menaçant et inaccessible. Il ne parait dans les rues qu'en voiture ou monté à cheval, entouré d'une effrayante escorte. [...] La concentration en ses mains du pouvoir judiciaire et administratif, son droit d'expulsion hors de Perse, réduit les sujets russes à l'impuissance. Le consul et la colonie sont deux mondes séparés, souvent en conflit ${ }^{36}$.»

Les attaques contre les consuls ne cessent pas avec le temps. L'été 1917, où la Russie s'enfonce dans le chaos politique, voit une recrudescence des diatribes à leur encontre. En septembre 1917, les Nouvelles du soviet de Qazvin comprennent un long article intitulé «Quelques mots sur notre corps diplomatique en Perse », qui s'en prend violemment au consul dans la ville, digne représentant de l'ancien régime et fidèle incarnation de ce que les auteurs appellent le «cloaque diplomatique ${ }^{37}$ ». Voilà une communauté qui, refusant d'écouter les arguments des consuls, rejette catégoriquement un corps sur lequel elle veut rejeter les fautes de l'ancien régime. Dès avril, le consul-général à Tabriz doit démissionner face à la pression des ressortissants russes ${ }^{38}$. À Khoy, le vice-consul Bravin défend pied à pied ses prérogatives face au comité exécutif révolutionnaire, en clamant que son «viceconsulat est un représentant et un porte-voix fidèle des préceptes du nouveau gouvernement russe ${ }^{39} »$. Les institutions centrales de Petrograd et de Tiflis se trouvent accablées par les télégrammes exposant le conflit entre Bravin et Bruevich, chef du soviet local ${ }^{40}$.

La conversion de l'affrontement révolutionnaire en opposition entre communautés et consuls alimente une dynamique, comme le montre le cas des Iraniens du Caucase russe. Très

Christian Windler, La Diplomatie comme expérience de l'autre: consuls français au Maghreb, 1700-1840, Genève, Droz, 2002, p. 82-83.

${ }^{36}$ STsSSA, f. 2081, op. 1, d. 192, 1. 82, rapport du soviet d'Enzeli, 4 mai 1917 (17 mai 1917).

${ }^{37}$ STsSSA, f. 2081, op. 1, d. 192, 1. 193, note du consulat à Qazvin, n. d. [septembre 1917].

${ }^{38}$ The National Archives (Londres, TNA), FO 248/1188, rapport du consul britannique Shipley, 19 avril 1917 ; pour une comparaison avec le cas des consuls contestés au Levant lors de la Révolution française, voir P. Even (dir.), Les Français à l'étranger..., op. cit., p. 182-185.

${ }^{39}$ Lettre du vice-consul Bravin au comité exécutif de Khoy, 18 mai 1917 (31 mai 1917), cité dans L. Sargizov, Assirijtsy stran Blizhnego, op. cit, p. 95-96; STsSSA, f. 2081, op. 1, d. 192, 1. 47-48, lettre de Bravin au fonctionnaire diplomatique à Tiflis, 24 avril 1917 (7 mai 1917).

${ }^{40}$ STsSSA, f. 2081, op. 1, d. 192, 1. 151, télégramme de Bravin au Troisième département du ministère des Affaires étrangères, 31 juillet 1917 (13 août 1917). 
nombreux, occupant des positions diversifiées parmi le prolétariat urbain, l'artisanat et les marchands de la région, les Iraniens du Caucase ont derrière eux une tradition de politisation qui remonte au moins à la révolution constitutionnelle persane de 1906 dont ils ont été un des moteurs. Les Iraniens de Tiflis, qui s’organisent politiquement dès avril 1917, obtiennent ainsi du soviet de la ville qu'il condamne les politiques tsaristes en Iran ${ }^{41}$. À Bakou, les travailleurs iraniens s'agitent et refusent aussi de reconnaître l'autorité de leur consul et de ses agents. Le jour de Novruz, les travailleurs font irruption dans l'école Ettehâd et leur délégué, Mirza Ghaffârzâde Ardabili, déclare :

«Puisque le consul a refusé de se pencher sur nos plaintes, nous avons déchu nous-mêmes l'agent consulaire de ses fonctions et boycottons le consulat. Nous ne voulons plus confier à d'autres personnes nos affaires ${ }^{42}$. »

Les marchands iraniens, qui ne sont pourtant pas enthousiasmés par l'orientation radicale du mouvement, passent finalement une alliance tactique avec les ouvriers contre le consul avec lequel ils ont aussi des différends.

Des assemblées mi-communautaires mi-révolutionnaires mettent au jour les prévarications du consul, accusé d'avoir détourné avec son adjoint les fonds de l'école Ettehâd ${ }^{43}$. Le consul Mirza Djavâd Khan est finalement poussé à la démission par les ouvriers et Sâ'ed ol-Vezara (ancien adjoint du consul-général à Tiflis) devient consul à sa place le 17 mai ${ }^{44}$. À la fin du mois de mai, la fondation du parti Adâlat, proche des sociaux-démocrates de Bakou mais composés de ressortissants iraniens, marque une tentative d'arrimage des événements persans à la révolution russe. Dans son journal, le Bayraq-e 'Adâlat, le parti se positionne à la fois sur

${ }^{41}$ Mais le soviet ne prend pas de résolution sur un retrait des troupes iraniennes. Voir Kâveh Bayât, Tûfân bar farâz-e Qafqâz, Téhéran, Markaz-e Asnâd va târîkh-e diplumâsî, 1380 (2001), p. 17-18.

${ }^{42}$ Rapport du consulat à Bakou au ministère des Affaires étrangères, 19 Radjab 1335 (11 mai 1917), cité par K. Bayât, Tûfân bar farâz-e Qafqâz, op. cit., p. 24-25 ; Ghaffârzâde est arrivé au Caucase en 1903 et devient un des dirigeants du jeune mouvement communiste, avant d'être assassiné au Gilan en 1918 : Touraj Atabaki, " Constitutionalism in Iran and its Asian Interdependencies », Comparative Studies of South Asia, Africa and the Middle East, 28 (1), 2008, p. 142-153, p. 149.

${ }^{43}$ Mohammad Khan Fahîmî, adjoint du consul, reconnaît le détournement de vingt-cinq mille roubles appartenant à l'école, pour le compte du consul. Mohammad Khosrowpenâh et Oliver Bast, Ferqa-ye 'Adâlat-e Irân az djanûb-e Qafqâz tâ Shimâl-e Khorasân, 1917-1920, Téhéran, Pardes Danish, 1388 (2009), p. $5-7$; sur la méfiance de la bourgeoisie marchande de Bakou envers le mouvement des travailleurs, voir les mémoires de Bahram Agazade, Internationaal Institut voor Sociale Geschiedenis (Amsterdam, IISG), ARCH-01405, mf. 1.

${ }^{44}$ Bâqer 'Âqelî (dir.), Khâterât-e siyâsî-e Mohammad Sâ'ed Marâghâ̂, Téhéran, Nâmak, 1373 (1994), p. 5960 ; Nezâm'ali Dahnavî, Irâniân mohâdjir dar Qafqaz : fa'âliyathâ-ye farhangî dar sâlhâ-ye 1900-1931, Téhéran, Merkez-e asnâd ve khidmât-e pazhuheshî, 1383 (2004), p. 40-41. 
les échiquiers politiques russe et iranien. En juin, il lance un appel aux forces démocratiques iraniennes, en vue de mettre en œuvre un programme révolutionnaire commun, mais n'obtient qu'un écho limité, témoignant des difficultés de la politisation entre les espaces impériaux ${ }^{45}$. Ces processus simultanés d'attaque contre les institutions consulaires s'appuient sur les caractéristiques structurelles d'une région marquée par le pouvoir et les abus des consuls, et laissent entrevoir la possibilité d'une communauté d'action qui transcende les espaces impériaux.

\section{La possibilité d'une révolution transnationale}

Le rejet par les communautés de leurs institutions consulaires est particulièrement important, car il marque la possibilité d'une révolution conjointe. Il distingue la situation en Iran de celle en Anatolie orientale, où la situation d'occupation maintient une forme de barrière entre citoyens russes et populations locales ${ }^{46}$. Le discours des révolutionnaires russes en Iran du Nord revient à redécouper les frontières sociopolitiques acceptées : en séparant les consuls de leur communauté, il ouvre la porte à une solidarisation entre sujets iraniens et russes, unis dans une même oppression infligée par les autorités tsaristes. Le bulletin de la Banque d'escompte de Tabriz note à la fin d'avril : « Le renversement et la chute de l'ancien régime en Russie se sont reflétés dans l'attitude des Persans, qui nous sont devenus beaucoup plus favorables ${ }^{47}$. »

Dans ce renversement, le souvenir des années 1900 occupe une place cruciale. Au cours des premiers jours de mars 1917, les révolutionnaires russes multiplient les commémorations aux victimes de la première révolution russe, comme le montre une autre photographie de Nino Djordjadze (document 2) ${ }^{48}$. Au même moment, la repolitisation des villes d'Iran du Nord suit l'héritage de la révolution constitutionnelle iranienne et des partis démocrates,

\footnotetext{
${ }^{45}$ M. Khosrowpenâh et O. Bast, Ferqa-ye 'Adâlat-e Irân, op. cit., 1388 (2009), p. 8-15 et 25 ; 1'Adâlat peut
} se nourrir du fort mécontentement de la communauté iranienne en raison des conditions matérielles dégradées : Ronald Grigor Suny, The Baku Commune, 1917-1918: Class and Nationality in the Russian Revolution, Princeton, Princeton University Press, 1972, p. 110-111; pour la vision soviétique, Tsatur Agajan, Vekovaja druzhba narodov Zakavkaz'ja, T. 2, Erevan, Ajastan, 1972, p. 22-23.

${ }^{46}$ Sur ce point, voir le protocole de la réunion des soviets du Lazistan, 26-27 août 1917 (STsSSA, f. 1850, op. 1, d. 6, 1. 5-6).

${ }^{47}$ Shevket Tagieva, Natsional'no-osvoboditel'noe dvizhenie v Iranskom Azerbajdzhane v 1917-1920 gg., Bakou, Izdatel'stvo AN ASSR, 1956, p. 35-36.

48 Tamar Lortkipanidze, Nino Djordjadze. Kartveli kalis tvalit danakhuli p’irveli msoplio omi, Tbilissi, Sezani, p. 139. 
comme le rappellent les historiens Ahmad Kasravî et 'Alî Azarî ${ }^{49}$. L'interprétation de ce passé, problématique en raison du rôle de l'armée tsariste dans l'écrasement de cette révolution en 1911-1912, devient un enjeu important dans la rencontre entre un mouvement révolutionnaire russe et des acteurs politiques locaux. Les démocrates iraniens de Tabriz, enhardis par la libéralisation politique qu'entraîne l'affaiblissement de la tutelle russe, prennent l'initiative de ce rapprochement mémoriel et des déclarations communes sont faites avec le comité exécutif russe élu à Tabriz ${ }^{50}$. Ils décident d'organiser, le 7 avril, une commémoration rappelant l'ouverture du premier parlement persan : au cours des années précédentes, la célébration du 7 avril était interdite par les autorités prorusses, mais les démocrates de Tabriz convient cette fois la communauté russe et les officiers de l'armée tsariste. Dans un rapport écrit au lendemain de la cérémonie, le consul français dépeint ces officiers arborant des cocardes rouges, dans un mélange de pardon public et de symbolique révolutionnaire ${ }^{51}$.

Ce premier événement est suivi, le 15 juin, par une véritable « cérémonie expiatoire », pour reprendre les mots du même consul. La cérémonie célèbre cette fois les morts de l'intervention russe, en décembre 1911, en particulier la figure du révolutionnaire Siqqet olEslâm, pendu dans le cadre de la sanglante répression des premiers jours ${ }^{52}$. Le consul de France, témoin de la scène, en fait le récit suivant :

«C'est surtout sur la tombe de Seqqat-el-Islam qu'a eu lieu la partie principale de la démonstration : un officier russe y a pris la parole pour excuser le peuple russe des actes de sauvagerie commis à Tauris par le tsarisme durant la révolution de 1911-1912 ; le Kargouzar ${ }^{53}$, un membre du comité exécutif russe, un orateur du club démocrate persan et un mollah ont également prononcé de brefs discours, tendant tous à faire appel à une union très étroite des socialistes persans et russes pour travailler au développement et à la consolidation dans les deux pays de l'œuvre révolutionnaire et sociale ${ }^{54}$. »

49 'Alî Azarî, Qiyâm-e Shaykh Mohammad dar Tabrîz, Téhéran, Bongâh-e Safî 'Alî Shâh, 1346 (1967), p. $92-94$.

${ }^{50}$ Ibid., p. 97-98.

${ }^{51}$ Archives du ministère des Affaires étrangères français (MAE), Nouvelle Série, Perse, vol. 11, f. 129-130, rapport du consul français à Tabriz au ministère des Affaires étrangères, 8 avril 1917.

52 Sur l'exécution sommaire des leaders constitutionnalistes en décembre 1911, voir J. D. Clark, «Constitutionalists and Cossacks... », art. cité, p. 222-223.

${ }^{53}$ Fonctionnaire du ministre des Affaires étrangères iranien, chargé dans les provinces des relations avec les ressortissants et représentants étrangers, ainsi que des questions frontalières. Voir les trois articles publiés à ce sujet par Vanessa Martin et Morteza Nouraei dans le Journal of the Royal Asiatic Society en 2005-2006.

${ }^{54}$ MAE, Nouvelle Série, Perse, vol. 11, f. 154-155, rapport du consul français à Tabriz au ministère des Affaires étrangères, 15 juin 1917 ; ‘A. Azarî, Qiyâm-e Shaykh Mohammad dar Tabrîz, op. cit., p. 99. 
Cette cérémonie apparaît, dans son personnel et ses déclarations, comme un point d'orgue pour l'idéal de fraternisation et de collaboration des forces révolutionnaires. La «fête révolutionnaire » prend ici l'apparence du dépassement d'un passé tragique ${ }^{55}$. Elle s'accompagne, dans d'autres localités du Nord-Ouest iranien, de cérémonies locales similaires, où des constitutionnalistes emprisonnés parfois depuis plusieurs années sont libérés ${ }^{56}$. Les révolutionnaires russes et persans y insistent sur la solidarité des despotismes, qui rend d'autant plus nécessaire une résistance transnationale. «Soutenu par le consul russe, le pouvoir provincial procède impunément à ses abus contre la population locale ${ }^{57}$ ", écrivent les Russes d'Anzali.

Le rapprochement de la révolution russe permet aux forces démocrates iraniennes de reconstituer des structures, à l'abri de la répression du pouvoir. En Azerbaïdjan iranien, le parti démocrate d'Azerbaïdjan (Firqeh-e demokrat-e Azerbayjan) relancé par le sheikh Khiyâbânî à partir du printemps 1917 devient une des forces majeures du libéralisme et du réformisme politique. Dans son premier numéro, le 9 avril 1917, l'organe officiel de ce parti insiste sur le bouleversement que constitue la révolution russe pour l'ensemble de la région. Reprenant certains arguments des révolutionnaires caucasiens, le parti examine la question des autonomies régionales comme préalable à une véritable démocratisation et à une souveraineté populaire ${ }^{58}$. Le parti démocrate joue un rôle central dans l'organisation des cérémonies d'avril et de juin 1917, comme le soulignent les rapports de l'administration persane qui suit avec inquiétude le renouveau d'une contestation politique qu'elle n'a pas les moyens de contrôler ${ }^{59}$. Dans son activité s'exprime le mélange d'inspirations de la révolution russe et du répertoire de la révolution constitutionnelle, comme lorsque les démocrates appellent au début de juillet à la reconstitution d'un andjoman régional d'Azerbaïdjan, destiné

${ }^{55}$ Sur les fêtes révolutionnaires en contexte étranger: C. Windler, La Diplomatie comme expérience de l'autre, op. cit., p. 196-201.

${ }^{56}$ C'est le cas à Kazvin, où le soviet russe libère des individus emprisonnés depuis 1913 : Godzhibaba Azimov, Velikij Oktjabr' v Azerbajdzhane, Bakou, Azerbajdzhanskoe Gosudarstvennoe Izdatel'stvo, 1987, p. 138 ; Olisöhbət Sumbatzadə (dir.), Cənubi Azərbaycan tarixinin oçerki (1828-1917), Bakou, Elm, 1985, p. 203.

${ }^{57}$ STsSSA, f. 2081, op. 1, d. 192, 1. 82, rapport du soviet d'Enzeli, 4 mai 1917 (17 mai 1917).

${ }^{58}$ Ervand Abrahamian, Iran Between Two Revolutions, Princeton, Princeton University Press, 1982, p. $112-$ 113.

${ }^{59}$ Rapport du gouverneur-général d'Azerbaïdjan au ministère de l'Intérieur, 25 Sha'bân 1335 (16 juin 1917), Kâveh Bayât (éd.), Irân ve djang-e djahânî-e avval : Âsnâd-e vezârat-e dâkhile, Téhéran, Intishârât-e Sâzemân-e Asnâd-e Melli-e Irân, 1381 (2002), p. 370-371 ; Manuchahr Hedâyatî Khûshkalâm (éd.), Yâddâshthâ-ye Âhmad Kasmâyî az nahzat-e Djangal, Rasht, Komite-ye Gil, 1383 (2004), p. 85 ; Sh. A. Tagieva, op. cit., p. $42-43$. 
à exprimer la souveraineté populaire face aux autorités gouvernementales ${ }^{60}$. Au-delà d'une certaine imitation et d'une solidarité sincère dans les premiers temps, les démocrates persans trouvent ainsi un allié commode pour rétablir des forces décimées au début des années 1910.

Les différents éléments qui indiquent une forme de politisation faisant fi des frontières étatiques et politiques officielles n'impliquent cependant pas une fluidité absolue. La fraternisation rendue possible par l'élan révolutionnaire ne signifie pas un oubli généralisé des griefs qu'entretiennent les Iraniens à l'égard des Russes. Observateurs et témoins rapportent avec un certain scepticisme la brusque fraternisation à laquelle prétendent les soldats russes en Perse. L'historien et témoin iranien Ahmad Kasravî résume ce scepticisme persistant quand il écrit :

«Partout les soldats et cosaques s'agitaient, fondaient des comités et manifestaient leur désir de liberté. Ils se rendirent chez les libéraux persans auxquels ils donnèrent du "frère" [qârdâsh] et du "camarade" [yoldâsh]. Ils étaient désormais des "camarades"! Ces "camarades" qui hier encore nous mettaient en joue et avaient fait couler à de nombreuses reprises le sang de notre jeunesse ! Où étaient-ils passés ces soldats qui hier chantaient dans les rues les hymnes impériaux et de leurs voix retentissantes faisaient frémir les cœurs ${ }^{61}$ ?»

Une partie significative de la population du Nord de l'Iran se tient ainsi à l'écart de la fraternisation proposée, aussi bien par hostilité aux idées révolutionnaires que par nationalisme. Le ministre de France à Téhéran évoque en mai le nouvel orgueil des Iraniens face aux « tyranneaux » de la veille, désormais « dégonflés ${ }^{62}$ ».

Signe de cette nouvelle assurance, les autorités iraniennes renvoient nombre des experts et conseillers russes placés en leur sein sous l'influence de la diplomatie tsariste, au motif d'y mettre en œuvre des réformes ${ }^{63}$. Cependant, les espoirs d'une transformation radicale de la politique russe en Perse ne se confirment pas et le retrait rapide de l'ancienne armée tsariste

\footnotetext{
60 'A. Azarî, Qiyâm-e Shaykh Mohammad dar Tabrîz, op. cit., p. 108-109 ; Ahmad Kasravî, Târîkh-e hidjdeh sâle-ye Âzarbâidjân : Bâzmânde-ye târîkh-e mashrûtê-ye Îrân, Téhéran, Amir Kabir, 1340 (1961), p. 674-678.

${ }^{61}$ A. Kasravî, Târîkh-e hidjdeh sâle-ye Âzarbâidjân, op. cit, p. 674-675 ; le diplomate allemand Wipert von Blücher fait preuve d'une ironie très similaire pour qualifier le zèle avec lequel les soldats russes se mettent à déclarer : «L’Ancien régime russe qui voulait opprimer la Perse est renversé. Le temps est désormais venu de l'amitié entre la Russie libre et la Perse libre. » (Wipert von Blücher, Zeitenwende in Iran : Erlebnisse und Beobachtungen, Biberach-an-der-Riss, Koehler \& Voigtländer, 1949, p. 117.)

${ }^{62}$ MAE, Nouvelle Série, Perse, vol. 11, f. 133-135, rapport du ministre français à Téhéran au ministère des Affaires étrangères, 2 mai 1917.

${ }^{63}$ STsSSA, f. 2081, op. 1, d. 192, 1. 259-260, lettre du citoyen russe Egan Bek Melik Eganov à Papadzhanov, 2 septembre 1917 (15 septembre 1917); 'Abdollah Bahrâmî, Khâterât-e 'Abdollah Bahrâmî, s.1., 1344 (1965), vol. 1 , p. 538-539.
} 
n'est pas mis en œuvre. Le gouvernement provisoire adopte une politique louvoyante sur le sujet et les attentes de la population persane ne sont finalement pas satisfaites ${ }^{64}$. Alors que février 1917 semblait promettre une refondation des relations diplomatiques entre les deux États, libérées du poids des militaires russes, l'occupation demeure une réalité douloureusement ressentie par le mouvement démocrate iranien. Faute d'égalité, la convergence des luttes révolutionnaires apparaît de plus en plus illusoire.

\section{Décomposition économique et tensions politiques}

La principale menace à l'existence d'un espace politique transfrontalier ne vient-elle cependant pas d'un autre côté, lié aux évolutions structurelles de l'espace sous domination russe ? Sur un plan politique, les revendications locales se multiplient et créent des forces centrifuges dans le territoire révolutionnaire. C'est ainsi que la révolution proprement dite passe au second plan dans les territoires d'Anatolie occupée, où le principal enjeu devient l'établissement d'un nouveau statut pour la région, en faveur duquel les élites arméniennes de Russie s'engagent. Le 26 avril 1917, le gouvernement provisoire adopte un décret où l'expression d'Arménie turque remplace celle de territoires de Turquie occupée. Le nouveau territoire est retiré du ressort caucasien et soumis directement à Petrograd ${ }^{65}$. Mais la rupture est aussi liée à la décomposition politique intérieure de la Russie. À l'été, le putsch de Kornilov coupe partiellement le Caucase du Sud de la Russie et incite les dirigeants des Soviets locaux à suivre une ligne politique de plus en plus autonome ${ }^{66}$. Ces troubles

${ }^{64}$ Saleh M. Aliev, « Significant Changes in Russia’s Expansionist Policy towards Iran 1906-1917 », in Oliver Bast (dir.), La Perse et la Grande Guerre, Téhéran, Institut français de recherche en Iran, 2002, p. 84-85 ; le 8 avril, le consul français à Tabriz parle des Persans qui « voient déjà les troupes de nos alliés retirées du Nord de leur patrie et le régime du bon plaisir ressusciter en Perse » (MAE, Nouvelle Série, Perse, vol. 11, f. 129-130, rapport du consul français à Tabriz au ministère des Affaires étrangères, 8 avril 1917).

${ }^{65}$ STsSSA, f. 2081, op. 1, d. 227, 1. 8, lettre d'Aver'janov à l'Ozakom, 7 juin 1917 (20 juin 1917) ; Armen Marukjan, Armjanskij vopros $i$ politika Rossii (1915-1917 gg), Erevan, MIG NAN RA, 2003, p. 339-341 ; Richard G. Hovannisian (dir.), « The Competition for Erzurum, 1914-1921 », in Richard G. Hovannisian (dir.), Armenian Karin/Erzurum, Costa Mesa, Mazda, 2003, p. 370.

${ }^{66}$ Protocole de session du Centre régional des soviets, 3 septembre 1917 (16 septembre 1917), et résolution du Central régional des soviets, 17 octobre 1917 (30 octobre 1917), dans S. E. Sef, Revoljutsija 1917..., op. cit., p. 216-217 et p. 219 ; sur le coup d'État avorté de Kornilov, Joshua A. Sanborn, Imperial Apocalypse : The Great War and the Destruction of the Russian Empire, Oxford, Oxford University Press, 2014, p. 220-222. 
intérieurs ont des répercussions directes sur la communication à travers la frontière, en raison des mesures de fermeture adoptées par le gouvernement provisoire au cours du putsch ${ }^{67}$.

Plus encore, des questions économiques accélèrent la montée de tensions entre citoyens russes et autres, persans ou ottomans. La crise économique de l'Empire russe se trouve en effet accrue par la révolution. Mauvaises récoltes, épidémies et épizooties se multiplient, provoquant des troubles dans les provinces d'Erevan et d'Elisavetpol ${ }^{68}$. Les territoires frontaliers d'Iran et de Turquie apparaissent dans un premier temps comme des bases d'approvisionnement, où l'armée russe procède à des achats massifs de viande et de céréales. Cependant, le gouvernement iranien s'inquiète des répercussions de tels achats, alors que la récolte en Perse s'avère très décevante ${ }^{69}$. Les marchands persans eux-mêmes rechignent à accepter les roubles offerts en paiement de ces achats, car la chute de cette monnaie déjà très dépréciée depuis le début de la guerre s'accélère. Face à ces réticences à accepter le rouble, le gouvernement provisoire et l'Ozakom commencent à envisager de recourir au troc et à autoriser de nouveau des exportations de certains produits interdits (par exemple le sucre) pour pouvoir acheter des céréales ${ }^{70}$. Sur le terrain, cette évolution entraîne des tensions incessantes entre soldats russes et marchands locaux autour de l'achat de produits de base que l'intendance russe ne parvient plus à fournir.

Dans le cas des territoires occupés d'Anatolie orientale, les archives des administrations locales révèlent la montée en puissance des questions d'approvisionnement à la fin du mois de mai. La pénurie en biens agricoles, qui pose à la fois la question du rapport villes-campagnes et de celui entre groupes ethniques, est traitée directement par le soviet de la localité d'Of, à une cinquantaine de kilomètres de Trabzon, qui appelle « les populations turque et grecque »

\footnotetext{
${ }^{67}$ Note de l'ambassade russe au ministère des Affaires étrangères iranien, 8 Shavvâl 1335 (28 juillet 1917), in Mohammad Nâdir Nasîrî Moqaddam (dir.), Gozide-ye asnâd-e Daryâ-e Khazar ve manâteq-e shimâlî-e Irân dar djang-e djahânî-e avval, Téhéran, Daftar-e Mutâl'ât-e siyâsî ve baynamalalî, 1384 (1995), p. 225.

68 Gohar Avagjan, «Mik'ael Papazhanjani gortsuneut'juně Andrkovkasjan hatuk komitei (Ozakom) kazmum », Banber Hajastani arkhivneri, 2, 2008, p. 213-224, p. 217 ; David Marshall Lang, A Modern History of Soviet Georgia, New York, Grove Press, 1962, p. 198-199 ; voir les rapports dans STsSSA, f. 2081, op. 1, d. 55 .

${ }^{69}$ TNA, Foreign Office (FO) 248/1188, rapport du consulat britannique à Tabriz, 16 août 1917 ; STsSSA, f. 2081, op. 1, d. 341, 1. 152, rapport de l'adjoint au commissaire frontalier avec la Perse, 30 avril 1917 (13 mai 1917).

${ }^{70}$ STsSSA, f. 2081, op. 1, d. 192, 1. 221-225, une commission spéciale est créée à Petrograd à la fin de l'été et une commission équivalente est établie auprès de l'Ozakom.
} 
à apporter au marché leurs produits ${ }^{71}$. Dans toute l'Anatolie orientale, on signale la recrudescence d'incidents entre populations locales et soldats russes, autour de la question des réquisitions décrétées par l'armée russe ${ }^{72}$. Au Lazistan, les musulmans se plaignent d'irruptions de soldats russes qui les dépouillent et se comportent de manière provocante dans les lieux communautaires musulmans ${ }^{73}$. Alors que des soldats russes s'amusent à singer le muezzin depuis le haut du minaret local, le soviet d'Of décide de faire placarder un avertissement :

«Le devoir sacré de tout conquérant humain est de respecter les mœurs du pays conquis. Maintenant que nous sommes à l'avant-garde des peuples libres, nous devons d'autant plus respecter les croyances et les mœurs de la population locale des territoires de Turquie occupée ${ }^{74}$.»

Tensions économiques et culturelles se mêlent de plus en plus clairement, dans un mouvement commun à l'ensemble du pourtour caucasien de la Russie.

À partir de l'été, la multiplication des incidents entre une armée russe en pleine décomposition et les populations civiles en Perse réduit rapidement les opportunités de croisement et de coopérations politiques. Au Gilan et en Azerbaïdjan iranien, les vols et violences se multiplient le long des routes où circulent les soldats russes ${ }^{75}$. Les questions économiques qui entourent l'effondrement prolongé du rouble contribuent fortement aux tensions entre les soldats russes et la population urbaine. Les marchands du Nord de l'Iran menacent de ne plus accepter les roubles, dont ils n'ont désormais guère usage en raison de la fermeture persistante de la frontière russe à l'échange de la plupart des produits dont la région a besoin. Les refus d'accepter les roubles, si ce n'est à des taux très dégradés, provoquent la colère des militaires russes cantonnés dans les villes d'Azerbaïdjan iranien, qui ne peuvent

\footnotetext{
${ }^{71}$ STsSSA, f. 1850, op. 1, d. 10, 1. 5, proclamation du soviet d'Of, fin du printemps 1917.

${ }^{72}$ Voir sur ce point la lettre envoyée à l'Ozakom par le délégué sur place du comité arménien de Moscou, le 16 mai 1917 (29 mai 1917) : Makich’ Arzumanjan, Hajastan 1914-1917, Erevan, Hajastan, 1969, p. 739 ; lettre du général Youdenitch à l'état-major central, 28 avril 1917 (11 mai 1917), in Nikolaj Kakurin (dir.), Razlozhenie armii v 1917 godu, Moscou-Leningrad, Gosizdatel'stvo, 1925, p. 14-15.

${ }^{73}$ STsSSA, f. 1850, op. 1, d. 10, 1. 25, décision du comité exécutif du soviet d’Of, 28 mai 1917.

${ }^{74}$ STsSSA, f. 1850, op. 1, d. 10, 1. 19, proclamation du comité exécutif du soviet d'Of, 16 mai 1917.

${ }^{75}$ Note du karguzar du Gilan au ministère des Affaires étrangères iranien, $1^{\mathrm{er}}$ Ramazan 1335 (21 juin 1917),

Mohammad Nâdir Nasîrî Moqaddam (éd.), Gozîde-ye âsnâd-e daryâ-e khazar ve manâteq-e shimâli-e Irân

dar djang-e djahânî-e avval, Téhéran, Daftar-e Mutâl'ât-e siyâsî ve baynalmalalî, 1384 (1995), p. 218-219.
} 
plus compter sur des augmentations de rattrapage sur leurs soldes ${ }^{76}$. Le 6 juillet, dans un événement à grand retentissement, les troupes casernées à Ourmiya mettent à sac le bazar de la ville, pillant les biens qu'on refuse de leur vendre et molestant les marchands, avant d'incendier l'édifice ${ }^{77}$. Scellant la fin d'une parenthèse politique, ce pillage marque le caractère illusoire d'un rapprochement permis par la ferveur révolutionnaire, mais contradictoire avec la poursuite d'un pouvoir russe fondé sur l'occupation militaire.

\section{[pour le maquettiste : laisser une blanche]}

L'événement révolutionnaire rend en apparence possible dans ses premiers instants une double abolition de frontières : entre territoires, en donnant lieu à un processus critique audelà des frontières politiques; entre populations, aussi, car c'est bien au-delà des seuls ressortissants russes que la révolution a ses protagonistes. Cette abolition est rendue possible par une interprétation commune du passé récent de la région, rejetant l'impérialisme de la politique russe et des représentants de son autorité. L'écho entre les révolutions des années 1900, qui ont une première fois créé une dynamique politique régionale, et celle de 1917, représente un des principaux vecteurs de solidarisation transfrontalière. Mais l'idée d'un processus révolutionnaire commun, passés les premiers moments, achoppe sur des différences majeures, à commencer par la question du retrait russe de l'Iran, de la politique envers les territoires occupés ou encore des relations économiques. Progressivement, ces questions cristallisent les atermoiements de la révolution russe et ses divisions internes. Sur le terrain, ces tensions se traduisent par une hostilité croissante des populations locales envers une armée russe en pleine déliquescence. La déception engendrée par cette possibilité avortée de Février doit être prise en compte pour expliquer l'écho que reçoit la révolution bolchevique

\footnotetext{
${ }^{76}$ Les projets de réévaluation des soldes défendus par Nikolaj Nikolaevitch à la fin de 1916 s'embourbent après avril 1917. Voir STsSSA, f. 2081, op. 1, d. 291, 1. 11-13, lettre du chef de l'approvisionnement du front caucasien à l’Ozakom, 24 mars 1917 (6 avril 1917).

${ }^{77}$ MAE, Nouvelle Série, Perse, vol. 11, f. 179-181, rapport du consul français à Tabriz au ministère des Affaires étrangères, 13 juillet 1917 ; note du ministère des Affaires étrangères iranien à l'ambassade russe, 9 Shavvâl 1335 (29 juillet 1917), Mohammad Nâdir Nasîrî Moqaddam (éd.), op.cit., Téhéran, Daftar-e Mutâl'âte siyâsî ve baynalmalalî, 1384 (1995), p. 227-228 ; on dispose sur ce point du témoignage du poète russe Viktor Shklovskij, qui est alors affecté à l'armée russe dans la ville : Viktor Shklovskij, Sentimental'noe puteshestvie, Moscou, Novosti, 1990, p. 104-109; des tentatives sont simultanément effectuées par les kârguzârs pour procéder à des médiations en rassemblant marchands persans et militaires russes : Kh. Ataev, Politicheskie $i$ torgovo-ekonomicheskie otnoshenija severo-vostochnogo Irana v Rossii v nachale XX veka (1900-1917 gg.), Askhabad, Ylym, 1989, p. 115.
} 
d'Octobre, dont les leaders font preuve vis-à-vis de l'Iran d'un volontarisme remarqué. Abolissant les traités inégaux, les capitulations, la dette cumulée de l'Iran et annonçant le retrait de l'armée d'occupation, le gouvernement de Lénine témoigne d'une nouvelle résolution qui semblait avoir déserté les représentants régionaux de la première révolution de 1917.

Étienne Forestier-Peyrat, Université de Valenciennes, Centre d'histoire de Sciences Po, 75006, Paris, France.

Ancien élève de l'École normale supérieure de la rue d'Ulm, agrégé et docteur en histoire, Étienne Forestier-Peyrat est attaché temporaire d'enseignement et de recherche (ATER) à l'Université de Valenciennes et chercheur associé au Centre d'histoire de Sciences Po. Après avoir soutenu une thèse de doctorat consacrée à l'histoire du Caucase entre Russie, Iran et Turquie au $20^{\mathrm{e}}$ siècle, il poursuit des recherches sur l'économie politique des dettes publiques dans les États communistes et sur l'histoire transnationale du fédéralisme pendant la guerre froide. (etienne.peyrat@sciencespo.fr) 